\title{
Bone Marrow-Derived Mesenchymal Stem Cells Modified with Akt1 Ameliorates Acute Liver GVHD
}

Lukun Zhou', Shuang Liu², Zhao Wang ${ }^{1}$, Jianfeng Yao', Wenbin Cao ${ }^{1}$, Shulian Chen', Wenjun Xie ${ }^{1}$, Shuqing Feng ${ }^{3}$, Yuanfu Xu', Tao Cheng ${ }^{1}$, Mingzhe Han ${ }^{1}$ and Sizhou Feng ${ }^{1 *}$

\begin{abstract}
Background: Liver injury associated with acute graft-versus-host disease (aGVHD) is a frequent and severe complication of hematopoietic stem cell transplantation and remains a major cause of transplant-related mortality. Bone marrow-derived mesenchymal stem cells (BM-MSCs) has been proposed as a potential therapeutic approach for aGVHD. However, the therapeutic effects are not always achieved. In this study, we genetically engineered C57BL/6 mouse BM-MSCs with AKT1 gene and tested whether AKT1-MSCs was superior to control MSCs (NullMSCs) for cell therapy of liver aGVHD.

Results: In vitro apoptosis analyses showed that, under both routine culture condition and high concentration interferon- $\gamma($ IFN- $\gamma)(100 \mathrm{ng} / \mathrm{mL})$ stimulation condition, AKT1-MSCs had a survival (anti-apoptotic) advantage compared to Null-MSCs. In vivo imaging showed that AKT1-MSCs had better homing capacity and longer persistence in injured liver compared to Null-MSCs. Most importantly, AKT1-MSCs demonstrated an enhanced immunomodulatory function by releasing more immunosuppressive cytokines, such as IL-10. Adoptive transfer of AKT1-MSCs mitigated the histopathological abnormalities of concanavalin A(ConA)-induced liver injury along with significantly lowered serum levels of ALT and AST. The attenuation of liver injury correlated with the decrease of TNF- $a$ and IFN- $\gamma$ both in liver tissue and in the serum.

Conclusions: In summary, BM-MSCs genetically modified with AKT1 has a survival advantage and an enhanced immunomodulatory function both in vitro and in vivo and thus demonstrates the therapeutic potential for prevention and amelioration of liver GVHD and other immunity-associated liver injuries.
\end{abstract}

Keywords: AKT1, mesenchymal stem cells (MSCs), Liver aGVHD, ConA-induced liver injury, Cell therapy

\section{Background}

Acute graft-versus-host disease (aGVHD) develops in a significant number of patients who receive allogeneic hematopoietic stem cell transplantation (allo-HSCT), and liver is one of the most commonly affected organs [1-3]. Liver aGVHD is caused by the generation of alloreactive $\mathrm{T}$ cells that migrate to liver and induce liver injury [4]. It is a major cause of complications and death in patients who receive allo-HSCT and thus effective

\footnotetext{
*Correspondence: doctor_szhfeng@163.com

${ }^{1}$ State Key Laboratory of Experimental Hematology, National Clinical Research Center for Blood Diseases, Institute of Hematology \& Blood Diseases Hospital, Chinese Academy of Medical Sciences \& Peking Union Medical College, 288 Nanjing Road, Tianjin, China

Full list of author information is available at the end of the article
}

therapeutic strategies are needed [2]. Currently, the standard first-line treatment for aGVHD is immunosuppressive drugs, such as methylprednisolone and cyclosporine A. However, these drugs are often associated with secondary infections and about half of the patients do not respond to these drugs. So far, no effective strategy for steroid-refractory aGVHD has been established and new therapeutic strategies for these patients remain to be developed [5].

MSCs is a very promising treatment for aGVHD due to its immunosuppressive function. Recent studies have shown its effectiveness in many cases of aGVHD, especially in steroid-refractory aGVHD. However, the therapeutic effects are not always achieved [6-10].

(c) The Author(s). 2019 Open Access This article is distributed under the terms of the Creative Commons Attribution 4.0 International License (http://creativecommons.org/licenses/by/4.0/), which permits unrestricted use, distribution, and reproduction in any medium, provided you give appropriate credit to the original author(s) and the source, provide a link to the Creative Commons license, and indicate if changes were made. The Creative Commons Public Domain Dedication waiver (http://creativecommons.org/publicdomain/zero/1.0/) applies to the data made available in this article, unless otherwise stated. 
Genetically manipulating MSCs in vitro has promising potential in improving MSCs' functions to maximize its treatment potential, as shown by several studies [11-15]. AKT1 is a serine/threonine kinase that plays a key role in the modulation of cell proliferation and survival. It is well known for its anti-apoptotic effects against a variety of situations including oxidative and osmotic stress, irradiation and ischemic shock [16]. Recent studies have demonstrated that AKT1 played a pivotal role in regulating MSCs migration and secretion of paracrine cytoprotective factors [17-19]. In addition, Mangi and colleagues reported that AKT1-overexpressed MSCs were more resistant to apoptosis and could better prevent cardiac remodeling and restore the performance of infarcted hearts after transplantation into the ischemic rat heart [20]. Further study revealed that enhanced paracrine action of AKT1-MSCs accounted for MSCs function improvement $[18,19]$.

The inflammatory microenvironment plays a crucial role in the activation of MSCs. IFN- $\gamma$, a potent proinflammatory cytokine produced by activated T-cells, NK cells, NKT cells and macrophages, has impacts on many properties of MSCs, such as cell proliferation, differentiation, apoptosis and senescence [21-23]. It is also an inducer of the chemokine secretion and adhesion molecule expression of MSCs, which partially accounts for MSCs immunosuppressive and tissue reparative function [24-26].
In this study, we overexpressed AKT1 in mouse BMMSCs and evaluated its role in regulating cell viability and paracrine function under IFN- $\gamma$-stimulated condition. For in vivo study, we used a ConA-induced liver injury model to imitate liver aGVHD as they are similar in terms of the hepatotoxic mechanism, as both are induced by polyclonally activating $\mathrm{T}$ cells. We aimed to investigate whether AKT1-MSCs was superior to control MSCs (Null-MSCs) in resistant to apoptosis and amelioration of immune-mediated hepatitis induced by ConA, as well as to ascertain the potential mechanisms of these effects.

\section{Results}

\section{MSCs Culture and Characterization}

MSCs isolated from C57/B6 mouse bone marrow were obtained from the Cyaen company. These cells could expand for up to 20 passages. We analyzed the third passage of MSCs for cell morphology and cell surface markers. As shown in Fig. 1a, MSCs morphology was similar to fibroblasts which were fusiform or irregular triangle shaped. These cells had ovoid nuclei and 2 to 3 cytoplasmic processes of various lengths. Phenotypic analysis by flow cytometry demonstrated that these cells were positive for MSC markers CD29, CD44, Sca-1 and negative for major histocompatibility complex II (MHC

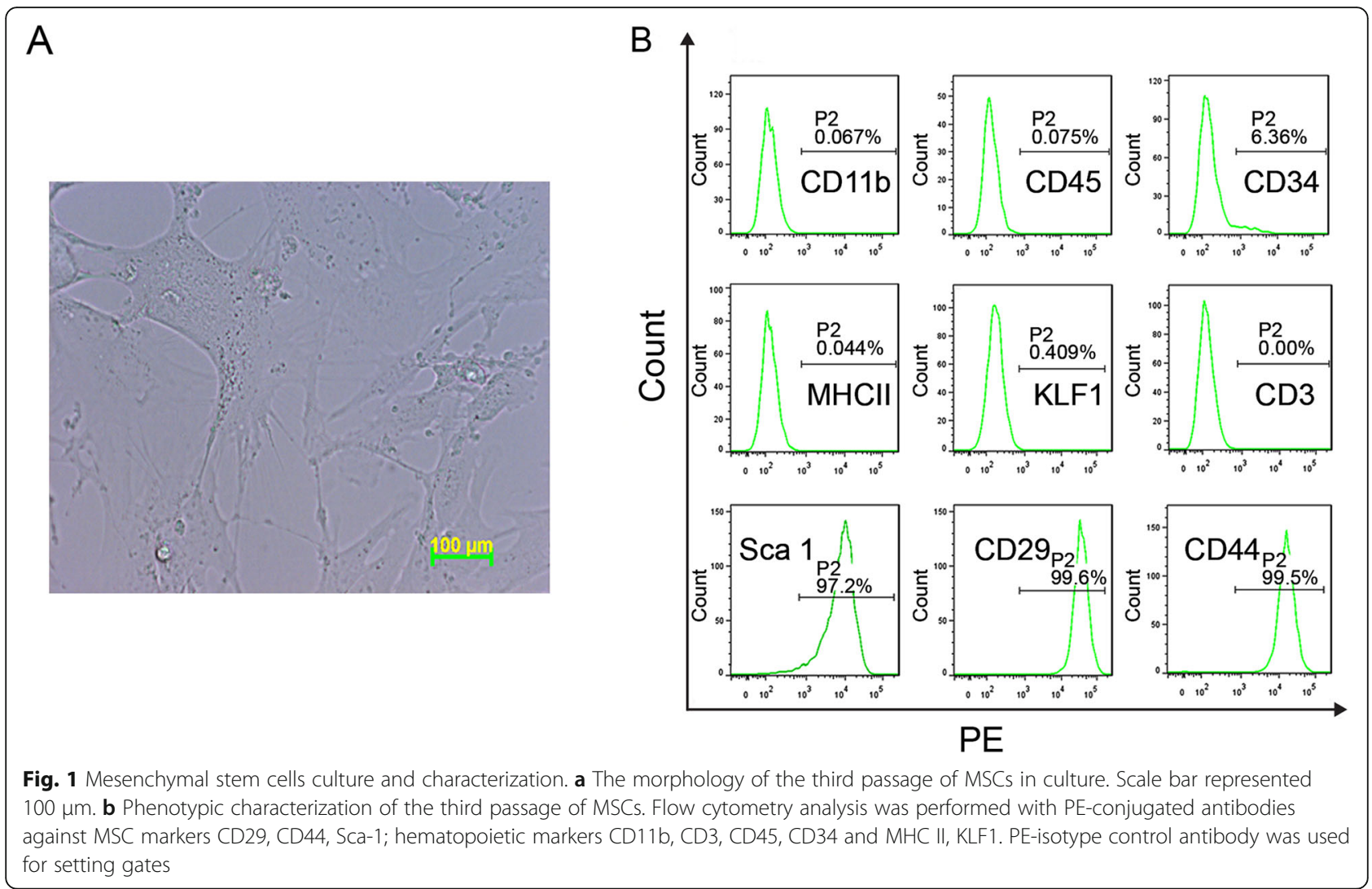


II), kruppel-like factor1 (KLF1) and hematopoietic markers CD11b, CD3, CD45 and CD34 (Fig. 1b).

\section{MSCs Genetically Modified with AKT1 Gene}

We used retroviruses to transduce MSCs with Null-GFP gene (Null-MSCs) and AKT1-GFP fusion gene (AKTMSCs), with over 95\% efficiency (Fig. 2a). The GFP+ cell population was flow-sorted and the expression of AKT1 was tested by real-time polymerase chain reaction (qRTPCR) and western blotting. qRT-PCR showed that AKT1 mRNA was about three-fold higher in AKT1MSCs compared with Null-MSCs (Fig. 2b). Overexpression of total AKT1 and phosphorylated AKT1 was further confirmed by Western blotting as shown in Fig. 2c. These data indicated successful incorporation of exogenous AKT1 gene into MSCs.

\section{Cytoprotective Effect of AKT1 Over-Expression on MSCs}

To test whether AKT1-engineered MSCs were more resistant to apoptosis than Null-MSCs, we performed apoptosis assessment of MSCs by Annexin V/PI staining. There was a slight decrease in apoptosis in AKT1-MSCs as compared with Null-MSCs under routine culture condition (Fig. 3a, b). We then investigated the survival capacity of MSCs under inflammatory circumstance. Null-MSCs and AKT1-MSCs were stimulated with IFN$\gamma(100 \mathrm{ng} / \mathrm{ml})$ for $24 \mathrm{~h}$, and then collected for apoptosis analysis. As shown in Fig. 3a, b, the percentage of apoptotic cells was significantly lower in the AKT1-MSCs

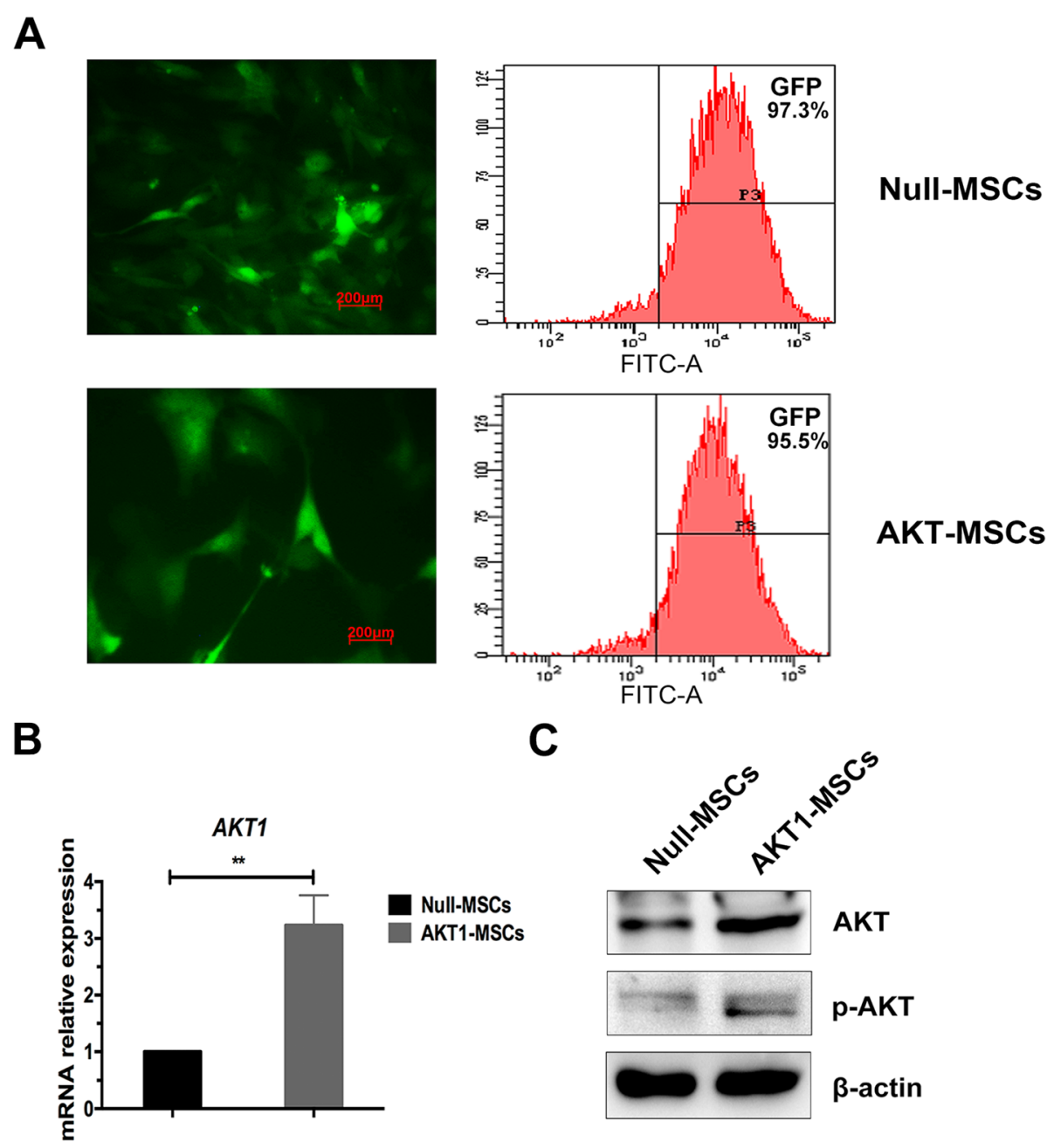

Fig. 2 Over-expression of AKT1 in MSCs. a At 72h after infection, the transduction efficiency of Null-MSCs and AKT1-MSCs was evaluated by fluorescence microscopy (left, Scale bar $=200 \mu \mathrm{m}$ ) and flow cytometry (right). Non-infected cells were used as the control for setting gates. b At $72 \mathrm{~h}$ after infection, the infected cells were sorted by flow cytometry for GFP+ population. The mRNA expression levels of AKT1 were quantified by qRT-PCR, normalized to those of GAPDH and set to 1 in Null-MSCs group. The data are presented as the means \pm SD of three independent experiments. ${ }^{*} P<0.05,{ }^{* *} P<0.01,{ }^{* * *} P<0.001,{ }^{* * *} P<0.0001$. C. Representative western blots showing total AKT1 and phosphorylated AKT1 expression in Null-MSCS and AKT1-MSCS 


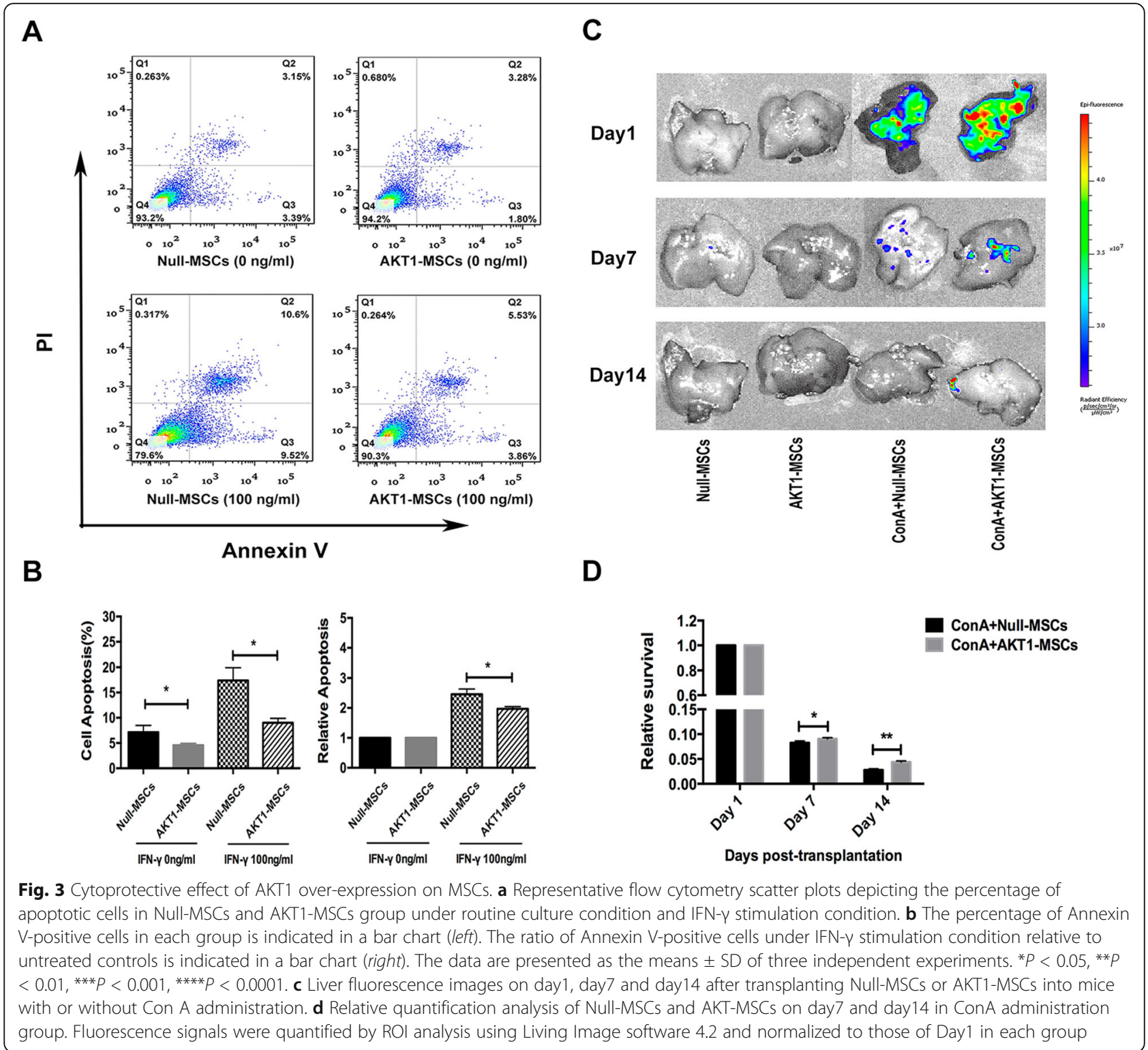

group than that in Null-MSCs group after IFN- $\gamma$ stimulation.

To further assess the cytoprotective effect of AKT1 over-expression on MSCs in vivo, we intravenously injected $5 \times 10^{6}$ Null-MSCs or AKT1-MSCs into mice with or without ConA administration, respectively. At day1 post-transplantation, a strong fluorescence signal was emitted from the liver in both Null-MSCs and AKT1-MSCs groups with ConA administration and no signal was detected in mice without ConA administration, suggesting the homing capability of MSCs to the damaged area. The fluorescence intensity was much stronger in AKT1-MSCs group than that in Null-MSCs group, which indicated that AKT1-MSCs have a better homing ability than Null-MSCs. At day 7 and day 14 after transplantation, though the fluorescence signal faded in both groups, it was still stronger in AKT1MSCs group than that in Null-MSCs group, suggesting AKT1-MSCs survived better than Null-MSCs in vivo (Fig. 3c, d).

\section{AKT1 Over-Expression Promoted Cytokines Release by MSCs}

It has been reported that MSCs has an immunosuppressive capacity, which is partially mediated by its secreted soluble factors, such as cytokines, chemokines and growth factors [27]. We investigated whether overexpression of AKT1 enhanced immunosuppressive cytokines release by MSCs under inflammatory environment. Null-MSCs and AKT1-MSCs were treated with IFN- $\gamma$ $(100 \mathrm{ng} / \mathrm{ml})$ for $12 \mathrm{~h}$ and then collected. qRT-PCR was performed to examine the mRNA expression of 
immunosuppressive cytokines interleukin-4 (IL-4), interleukin-10 (IL-10) and prostaglandin E synthase 2 (PTGES2). As shown in Fig. 4a, the mRNA of IL-4, IL10 and PTGES2 were significantly upregulated in AKT1MSCs compared to Null-MSCs. Interestingly, we also found the upregulation of hepatocyte growth factor (HGF) and vascular endothelial growth factor (VEGF) in AKT1-MSCs compared to Null-MSCs. These results were further confirmed at protein level by measuring cytokines in cell culture supernatant with ELISA. AKT1MSCs had a higher level of IL-10, HGF and VEGF after simulation with different dosages of IFN- $\gamma$ for $24 \mathrm{~h}$ (Fig. $4 \mathrm{~b})$. The above results suggested that AKT1-MSCs could not only release more anti-inflammatory cytokines (IL10) but also more growth factors (HGF, VEGF) under inflammatory environment, which contributed to its protective effect in immune-mediated hepatitis.

\section{AKT1-MSCs Was Superior to Null-MSCs in Ameliorating ConA-Induced Liver Injury}

To investigate the role of MSCs in liver aGVHD, we use a ConA-induced liver injury model to imitate liver aGVHD as they both closely related to $\mathrm{T}$ cell activation and pro-inflammatory cytokines release in terms of hepatotoxic mechanism. ConA $(20 \mathrm{mg} / \mathrm{kg})$ was injected intravenously into mice to induce liver injury and $5 \times 10^{6}$ Null-MSCs or AKT1-MSCs was injected into recipient mice $30 \mathrm{~min}$ after ConA administration. We examined the influences of Null-MSCs and AKT1-MSCs on liver injury by evaluating serum transaminase levels and liver tissue sections hematoxylin-eosin $(\mathrm{H} \& \mathrm{E})$. Serum transaminase assay revealed a significant decrease in alanine aminotransferase (ALT) and aspartate transaminase (AST) after MSCs injection, and there was more in mice received AKT1-MSCs (Fig. 5a). The protective effect of AKT1-MSCs was further confirmed by histopathology, which showed that hepatocellular injuries in AKT1MSCs recipients were less severe than those in NullMSCs recipients, as demonstrated by a significant reduction in the severity of tissue necrosis (Fig. 5b).

To further elucidate the molecular mechanism underlying the protective effect of MSCs on ConA-induced liver injury, cytokine expression was measured in serum and liver tissue at various time points after injection of Null-MSCs and AKT1-MSCs. As shown in Fig. 5c, administration of MSCs significantly reduced serum level of TNF- $\alpha$ and there was an additional and significant reduction of TNF- $\alpha$ in AKT1-MSCs recipients compared to Null-MSCs recipients. Null-MSCs did not reduce serum level of IFN- $\gamma$, whereas AKT1-MSCs was associated with a significant reduction of IFN- $\gamma$ (Fig. 5c). Furthermore, MSCs decreased ConA-induced proinflammatory cytokine gene expression in liver tissues as demonstrated by immunohistochemical staining of
TNF- $\alpha$ and INF- $\gamma$. There was an intense staining pattern of TNF- $\alpha$ and INF- $\gamma$ in ConA-treated mice without MSCs administration. However, in MSCs injection groups, TNF- $\alpha$ and INF- $\gamma$ staining levels were much lower and the expressions were even lower in AKT1MSCs injected mice compared with Null-MSCs injected mice (Fig. 5d, e). Since ConA is known to cause acute liver failure via an immunologic activated cytokine response syndrome with TNF- $\alpha$ and INF- $\gamma$ as the pivotal mediators, these data implied that AKT1-MSCs had a superior protective effect on immune-mediated hepatitis.

\section{Discussion}

MSCs derived from bone marrow may include HSCs and multipotent progenitor cells. In this study, we performed phenotypic analysis of the BM-MSCs by flow cytometry to determine its type and purity. As shown in Fig. 1b, these cells barely expressed hematopoietic cell surface markers such as CD34 (HSCs), CD45 (leukocytes), CD11b (monocytes/macrophages) and CD3 (lymphocytes), but they expressed MSCs specific markers CD29 and CD44, which confirmed its identity.

BM-MSCs has benefited a significant number of patients suffering from aGVHD. However, the therapeutic effects are not always reliably achieved [6-10]. Exploring new ways to improve its therapeutic efficiency would provide great clinical benefits. In this study, we genetically modified mouse BM-MSCs with AKT1 gene and evaluated its function. We used a ConA-induced liver injury model to mimic liver aGVHD as they are similar in terms of the hepatotoxic mechanism. ConA-induced hepatitis depends on the activation of $\mathrm{T}$ cells by macrophages in the presence of ConA, followed by the release of a variety of cytokines such as TNF- $\alpha$ and IFN- $\gamma$ to mediate inflammatory liver damage [28-30]. Liver aGVHD is caused by mature donor $\mathrm{T}$ cells that recognize alloantigens presented by host antigenpresenting cells (APCs), followed by a rapid burst of Tlymphocyte proliferation and tissue damage induced by activated $\mathrm{T}$ lymphocytes, cytokines and cells of the innate immune system [4]. They are both T-cell dependent liver injury disease. IFN- $\gamma$, a potent proinflammatory cytokine produced by activated T-cells, NK cells, NK/T cells and macrophages, is considered as a major pathogenic factor related to both ConA-induced liver injury and liver aGVHD [4, 22]. Thus, we compared cell apoptosis and the paracrine function of Null-MSCs and AKT1-MSCs under IFN- $\gamma$ stimulation condition. As shown in Fig. 3a, b, cell apoptosis was significantly reduced in AKT1-MSCs group as compared to Null-MSCs group. AKT1-MSCs also produced more immunosuppressive factors (e.g. IL-10) and growth factors (e.g., HGF and VEGF) critical for MSC-mediated tissue repair (Fig. 4a, b). 


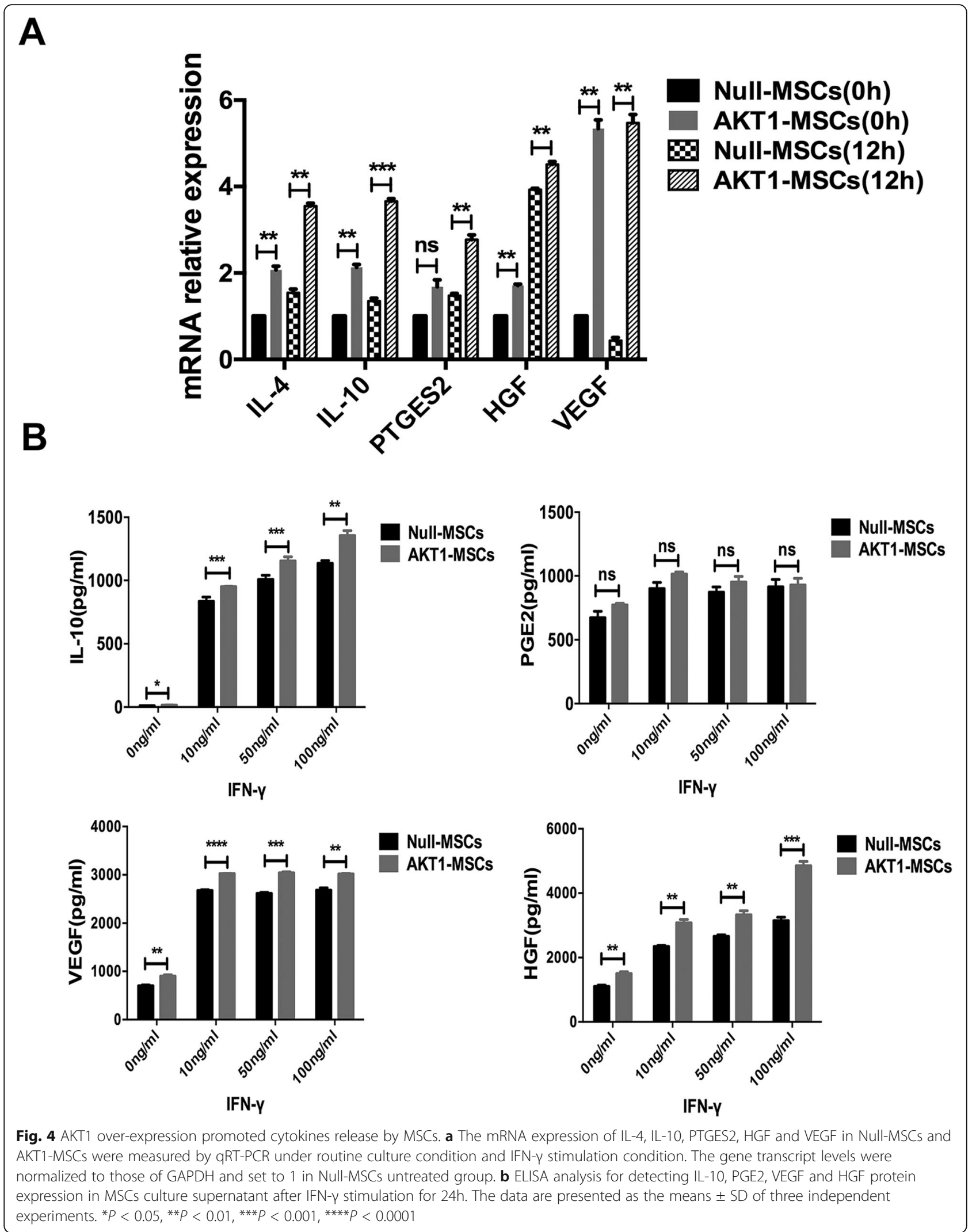


A

B
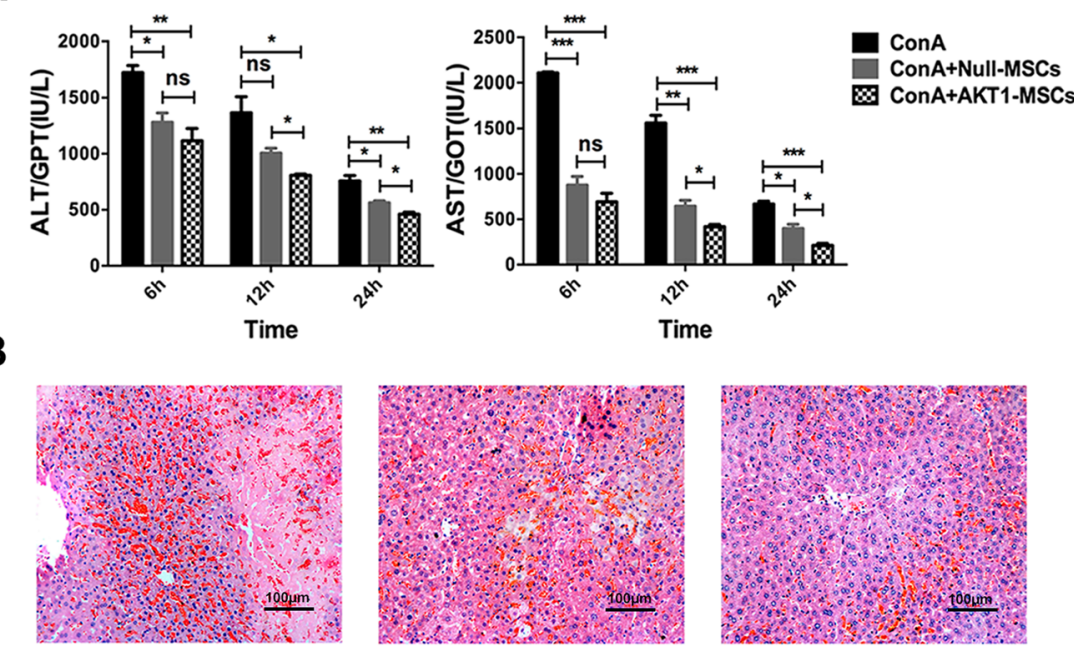

ConA

ConA+Null-MSCs

ConA+AKT1-MSCs

C
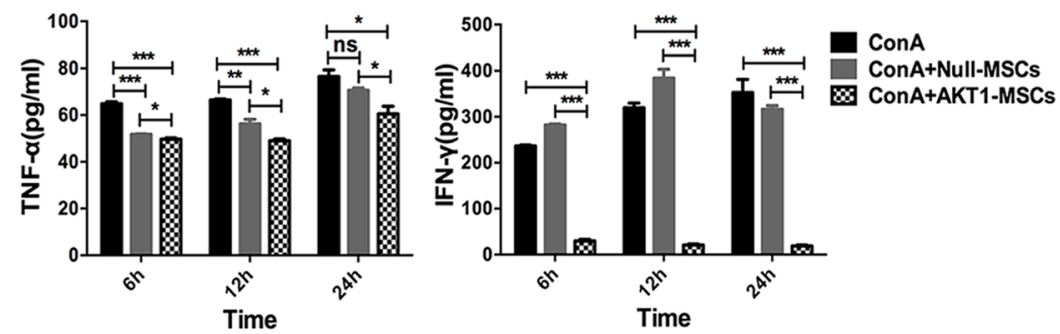

D
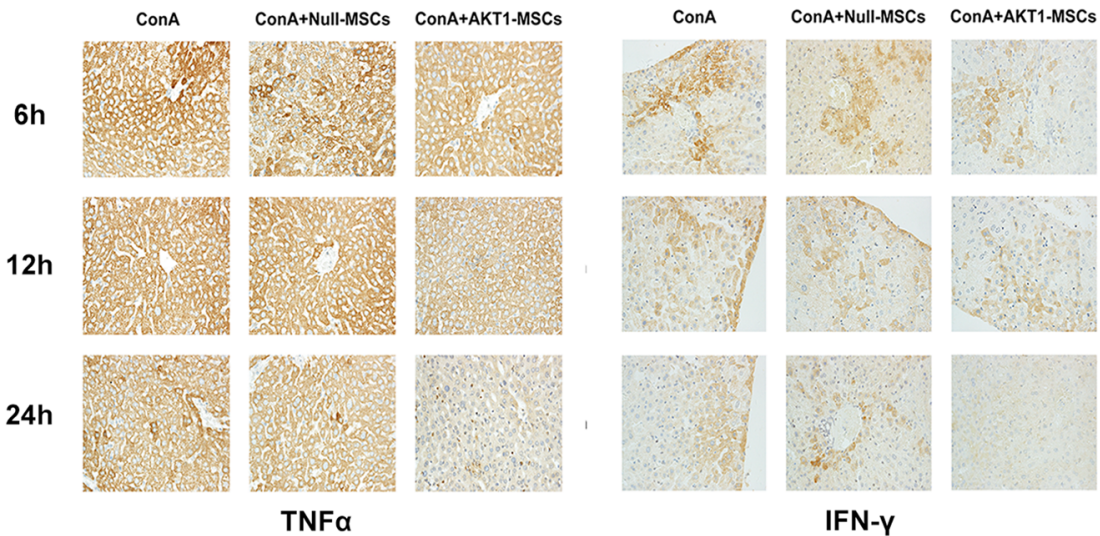

E
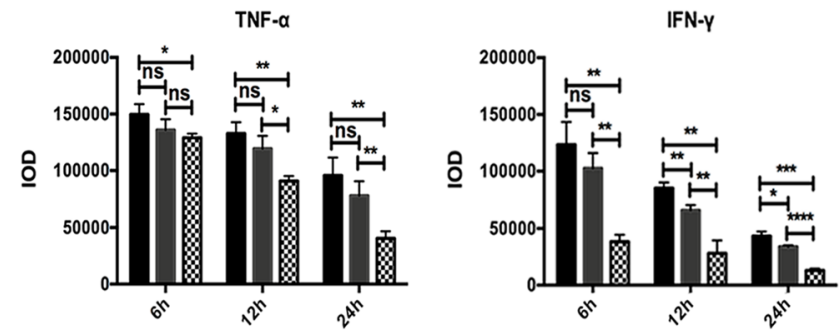

Fig. 5 (See legend on next page.) 
(See figure on previous page.)

Fig. 5 AKT1-MSCs ameliorated ConA-induced liver injury. Female C57BL/6 mice were intravenously injected with PBS (negative control), Null-MSCS $\left(5 \times 10^{6}\right)$, or AKT1-MSCs $\left(5 \times 10^{6}\right) 30 \mathrm{~min}$ after ConA $(20 \mathrm{mg} / \mathrm{kg})$ administration. a Serum levels of ALT and AST were measured at $6 \mathrm{~h}, 12 \mathrm{~h}$, and $24 \mathrm{~h}$ after MSCs injection. b Representative photographs of H\&E-stained liver tissue in each group were shown. Scale bar $=100 \mu m$. $\mathbf{c}$ The expression of TNF-a and INF- $\gamma$ in serum was measured by ELISA at $6 \mathrm{~h}, 12 \mathrm{~h}$ and $24 \mathrm{~h}$ after MSCs injection. $\mathbf{d}$ Intrahepatic TNF- $a$ and INF- $\gamma$ protein levels were measured by immunohistochemical staining at $6 \mathrm{~h}, 12 \mathrm{~h}$ and $24 \mathrm{~h}$ after MSCs injection. e The expression quantifications of TNF- $\alpha$ and INF- $\gamma$ were analyzed by ImagePro Plus 6.0. IOD (Integrated Option Density) was checked out by calculating the area and the density of the brown color in IHC staining. The data are presented as the means \pm SD of three independent experiments. ${ }^{*} P<0.05,{ }^{* *} P<0.01,{ }^{* * *} P<0.001,{ }^{* * *} P<0.0001$

In vivo studies showed that both Null-MSCs and AKT1-MSCs homed to the damaged liver section in ConA-administrated mice, but not to the normal liver section in PBS-administrated mice, suggesting that the injected MSCs preferably migrated to injured tissue (Fig. 3c). This is consistent with a previous study showing that MSCs preferentially homed to the site of myocardial ischemia but not to normal uninjured myocardium [20]. Interestingly, we also found that AKT1-MSCs has a homing advantage over Null-MSCs, as there was more AKT1-MSCs than Null-MSCs in liver section on day 1 after MSCs administration (Fig. 3c). It has been reported that several adhesion molecules and chemokines were involved in the homing process of MSCs, such as chemokine receptor type 4 (CXCR4), vascular cell adhesion molecule 1 (VCAM-1) and integrin $\beta 1$ [14, 31]. Further experiments need to be done to elucidate the mechanism of the improved homing ability of AKT1-MSCs. The homed MSCs survived longer in AKT1-MSCs group than in Null-MSCs group, which is in keeping with in vitro study showing AKT1 over-expression endowed BM-MSCs with a survival advantage.

The liver function of the AKT1-MSCs administered mice was significantly improved compared to the NullMSCs administered mice. This conclusion was based on the measurements of serum ALT and AST (Fig. 5a) and histological staining of liver tissue sections (Fig. 5b). More importantly, the expression of IFN- $\gamma$, which plays a pivotal role in liver inflammatory reaction, was also significantly reduced in AKT1-MSCs group, suggesting AKT1-MSCs was more effective than Null-MSCs in rescuing liver injury (Fig. 5c-e).

\section{Conclusions}

In summary, the results presented here showed that AKT1-modified BM-MSCs had a survival advantage and an enhanced immunomodulatory function compared to unmodified BM-MSCs, leading to better amelioration of ConA-induced liver injury. We further demonstrated that AKT1-modified BM-MSCs exerted a superior therapeutic effect through paracrine action by secreting more immunosuppressive factors and growth factors. The genetically modified MSCs with AKT1 could be used to prevent and ameliorate liver aGVHD after hematopoietic stem cell transplantation and other immunity-associated liver injuries. Clinical studies are needed to assess its therapeutic potentials.

\section{Methods \\ MSCs Culture and Identification}

Mouse (C57/B6) bone marrow MSCs was obtained from the Cyaen company (Guangzhou, China). MSCs was cultured in Dulbecco's Modified Eagle Medium/Nutrient Mixture F-12 (DMEM/F-12, GibcoTM, USA) supplemented with 10\% MSC-qualified Fetal Bovine Serum (GibcoTM, USA), 1\% penicillin/streptomycin (GibcoTM, USA), and 1\% GlutaMAX ${ }^{\mathrm{mi}}$-1 Supplement (GibcoTM, USA). At $80-90 \%$ confluency, cells were detached by trypsin/EDTA and passaged at a split ratio of 1:3. After detachment, cells were collected and incubated with the following phycoerythrin-conjugated antibodies: CD29, CD44, Sca-1, CD11b, CD3, CD45, CD34, MHC II and KLF1. The percentage of fluorescence-highlighted cells was determined by flow cytometry (Canto II, BD, USA).

\section{Generation of AKT1 Over-Expressing MSCs}

pMSCV-MCS-IRES-eGFP and pMSCV-AKT1-IRESeGFP, provided by Victor J Dzau's laboratory (Department of Medicine, Duke University School of Medicine), were co-transfected with packaging vectors VSVG and pKAT into HEK293T cells using lipofectamine 2000 (Invitrogen, USA) to produce retroviruses [20]. At $48 \mathrm{~h}$ and $72 \mathrm{~h}$ after transfection, viral supernatants were collected and concentrated to 100 -fold by ultracentrifugation after pelleting cell debris. AKT1 and control viruses were transduced into MSCs following a modified protocol reported by Rick et al [32]. In brief, immediately after detachment by trypsin, MSCs at passage 3 was mixed with virus at a multiplicity of infection (MOI) of 40 for $16 \mathrm{~h}$ in $0.5 \mathrm{ml}$ complete medium in the presence of 8 $\mu \mathrm{g} / \mathrm{ml}$ polybrene (Sigma, USA), followed by replacement with fresh complete medium. This procedure was repeated twice and the cells were expanded thereafter. GFP expression was visualized with a Zeiss Axio A1 fluorescence microscope. Successfully transduced MSCs was sorted by BD FACS Aria III System for GFP+ cells and expanded for another two passages before being used for the experiments. 
RNA Isolation and Real-Time Quantitative PCR (qRT-PCR) RNA was extracted with TRIzol reagent (Invitrogen, USA) and reverse-transcribed into cDNA using MMLV Reverse Transcriptase (Life Technologies, USA). qRT-PCR was performed on a 7500 Real-Time PCR system (Applied Biosystems, USA) with SYBR Green PCR kit (Takara, Japan) following the manufacturer's instructions. Primers used for PCR were listed as follows: (1) AKT1 5'-AACGGACTTCGGGCTGTG-3' and 5'-TTGTCCTCCAGCACCTCAGG-3'; (2) HGF 5'-ATCCACGATGTTCATGAGAG-3' and 5'-GCTGAC TGCATTTCTCATTC-3'; (3) VEGF 5'-GCGGGCTGCC TCGCAGTC-3' and 5'-TCACCGCCTTGGCTTGTCAC-3'; (4) IL-4 5'-AGAGATCATCGGCATTTTGAACGA-3' and 5'- CGAGCTCACTCTCTGTGGTGT TCT-3'; (5) IL-10 5'CTTGCACTACCAAAGCCACA-3' and 5'-GTTATTGTCT TCCCGGC TGT-3'; (6) PTGES2 5'-ACTTCCACTCCCTG CCCT-3' and 5'-CAGGTACCAAGGCTGGAT GT-3', respectively.

\section{Western Blot Assay}

Western blot assay was performed as previously described [33] with primary antibodies targeting AKT (Cell Signaling Technology, USA) and p-AKT (Cell Signaling Technology, USA). $\beta$-actin (Abcam, USA) was used as internal control.

\section{Apoptosis Assessment}

$5 \times 10^{5}$ cells were collected and stained with Annexin VAlexa Fluor 647-A and PI (BioLegend, USA) according to the manufacturer's instructions. Apoptosis assay was performed with flow cytometry (LSRII, BD, USA) and Annexin V-positive cells were identified as apoptotic cells.

\section{ConA-Induced Liver Injury Mouse Model and MSCs Transplantation}

To establish ConA-induced liver injury mouse model, 30 female BALB/c (H2d) mice (6 8 week, weighing 18-20 g) were randomly divided into three groups and $20 \mathrm{mg} /$ $\mathrm{kg}$ ConA was injected intravenously. At 30 minutes after ConA administration, mice in group $A(n=10)$ were injected with PBS via the tail vein. Group B $(n=10)$ received $5 \times 10^{6}$ Null-MSCs and Group $C(\mathrm{n}=10)$ received $5 \times 10^{6}$ AKT1-MSCs via the tail vein. The extent of liver injury was evaluated by serum transaminase assay and histopathological examination of liver tissue samples taken from sacrificed mice. All animal experiments were approved by the Institutional Animal Care and Use Committee of Peking Union Medical College.

\section{Histopathological Analysis, Immunohistochemistry and Serum Transaminase Assay}

At the time of sacrifice, liver tissue samples were collected, fixed in $4 \%$ formaldehyde for $24 \mathrm{~h}$ and embedded in paraffin. Then 3- $\mu \mathrm{m}$ liver sections were deparaffinized and fixed. Section samples were stained with hematoxylin solution (Sigma-Aldrich, Germany) for 5 min followed by eosin for $5 \mathrm{~min}$. TNF- $\alpha$ and IFN- $\gamma$ in liver sections were detected by immunohistochemistry (BOSTER.Bio, China) according to the manufacturer's instructions. For serum transaminase assay, serum ALT and AST levels were measured with an automated biochemical analyzer (Roche Integra 800, USA).

\section{Enzyme-Linked Immunosorbent Assay}

TNF- $\alpha$, INF- $\gamma$, IL-10, HGF, VEGF and PGE2 in serum and MSCs culture medium were measured using enzyme-linked immunosorbent assay (ELISA) kits (Liankebio, China) according to the manufacturer's instructions.

\section{In Vivo Fluorescent Imaging}

Null-MSCs and AKT1-MSCs were intravenously injected into the tail veins of BALB/c mice $30 \mathrm{~min}$ after ConA administration. Twenty-four hours after ConA injection, mice were euthanized and the livers were extracted. Tracking of in vivo transplanted MSCs localized to liver was performed using an IVIS fluorescence imaging system (Xenogen IVIS-200 Optical in Vivo Imaging System, Caliper Life Sciences Inc, Hopkinton, MA).

\section{Statistical Analysis}

Results were calculated as means $\pm \mathrm{SD}$. Statistical significance was evaluated with an unpaired Student's t-test and $P<0.05$ was considered statistically significant.

\section{Abbreviations \\ aGVHD: Acute graft-versus-host disease; Allo-HSCT: Allogeneic hematopoietic stem cell transplantation; ALT: Alanine aminotransferase; APCs: Antigen- presenting cells; AST: Aspartate transaminase; BM-MSCs: Bone marrow- derived mesenchymal stem cells; ConA: Concanavalin A; CXCR4: Chemokine receptor type 4; H\&E: Hematoxylin-eosin; HGF: Hepatocyte growth factor; IFN-ץ: Interferon-ү; IL-10: Interleukin-10; IL-4: Interleukin-4; KLF1: Kruppel-like factor1; MHC II: Major histocompatibility complex II; mRNA: Messenger ribonucleic acid; MSCs: Mesenchymal stem cells; PGE-2: Prostaglandin E2; PTGES2: Prostaglandin E synthase 2; qRT-PCR: Real-time polymerase chain reaction; TNF-a: Tumor necrosis factor a; VCAM-1: Vascular cell adhesion molecule 1; VEGF: Vascular endothelial growth factor}

\section{Acknowledgements \\ The authors would like to thank Chuanyi Mark Lu and Ping Zhang for their valuable suggestions on this manuscript.}

\section{Authors' contributions}

Lukun Zhou, Sizhou Feng, Mingzhe Han, Yuanfu Xu, Tao Cheng conceived the project, designed the study. Lukun Zhou and Shuang Liu performed experiments, analyzed data and prepared the manuscript. Zhao Wang, Jianfeng Yao, Wenbin Cao, Shulian Chen, Wenjun Xie, Shuqing Feng, performed some experiments and edited the manuscript. All authors read and approved the final manuscript.

\section{Funding}

This work was supported by grants from the National Megaproject on Key Infectious Diseases (2017ZX10202102), the CAMS Initiative for Innovative 
Medicine (2016-12M-1-017) and the Natural Science Foundation of Tianjin City (18JCZDJC34400).

\section{Availability of data and materials}

Datasets and materials are available by the corresponding author.

\section{Ethics approval and consent to participate}

All animal experiments were approved by the Animal Ethics Committee of Institute of Hematology and Blood Disease Hospital, Chinese Academy of Medical Sciences.

\section{Consent for publication}

Not applicable.

\section{Competing interests}

The authors declare that they have no competing interests.

\section{Author details}

'State Key Laboratory of Experimental Hematology, National Clinical Research Center for Blood Diseases, Institute of Hematology \& Blood Diseases Hospital, Chinese Academy of Medical Sciences \& Peking Union Medical College, 288 Nanjing Road, Tianjin, China. ${ }^{2}$ Guangzhou Medical University, 195 Dongfeng Xi Road, Guangzhou, Guangdong Province, China. ${ }^{3}$ North China University of Science and Technology Affiliated Hospital, 73, Construction South Road, Lubei District, Tangshan, Hebei Province, China.

\section{Received: 24 March 2019 Accepted: 22 November 2019}

\section{Published online: 16 December 2019}

\section{References}

1. Blazar BR, Murphy WJ, Abedi M. Advances in graft-versus-host disease biology and therapy. Nat Rev Immunol. 2012;12(6):443-58.

2. El-Sayed MH, El-Haddad A, Fahmy OA, et al. Liver disease is a major cause of mortality following allogeneic bone-marrow transplantation. Eur J Gastroenterol Hepatol. 2004:16(12):1347-54.

3. Salomao M, Dorritie K, Mapara MY, et al. Histopathology of graft-vs-host disease of gastrointestinal tract and liver: an update. Am J Clin Pathol. 2016: 145(5):591-603.

4. Ferrara JL, Levy R, Chao NJ. Pathophysiologic mechanisms of acute graft-vs.host disease. Biol Blood Marrow Transplant. 1999;5(6):347-56.

5. Martin PJ, Rizzo JD, Wingard JR, et al. First- and second-line systemic treatment of acute graft-versus-host disease: recommendations of the American Society of Blood and Marrow Transplantation. Biol Blood Marrow Transplant. 2012;18(8):1150-63.

6. Resnick IB, Barkats C, Shapira MY, et al. Treatment of severe steroid resistant acute GVHD with mesenchymal stromal cells (MSC). Am J Blood Res. 2013; 3(3):225-38

7. Zhao K, Lou R, Huang $F$, et al. Immunomodulation effects of mesenchymal stromal cells on acute graft-versus-host disease after hematopoietic stem cell transplantation. Biol Blood Marrow Transplant. 2015;21(1):97-104.

8. Le Blanc K, Rasmusson I, Sundberg B, et al. Treatment of severe acute graftversus-host disease with third party haploidentical mesenchymal stem cells. Lancet. 2004:363(9419):1439-41.

9. Ringden O, Uzunel M, Rasmusson I, et al. Mesenchymal stem cells for treatment of therapy-resistant graft-versus-host disease. Transplantation. 2006;81(10):1390-7.

10. Le Blanc K, Frassoni F, Ball L, et al. Mesenchymal stem cells for treatment of steroid-resistant, severe, acute graft-versus-host disease: a phase II study. Lancet. 2008:371(9624):1579-86.

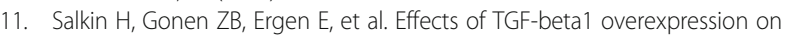
biological characteristics of human dental pulp-derived mesenchymal stromal cells. Int J Stem Cells. 2019;12(1):170-82.

12. Khalid RS, Khan I, Zaidi MB, et al. IL-7 over-expression enhances therapeutic potential of rat bone marrow mesenchymal stem cells for diabetic wounds. 2019;27(3):235-48

13. Choi JS, Jeong IS, Han JH, et al. IL-10-secreting human MSCs generated by TALEN gene editing ameliorate liver fibrosis through enhanced antifibrotic activity. Biomater Sci. 2019;7(3):1078-87.

14. Ma HC, Shi XL, Ren HZ, et al. Targeted migration of mesenchymal stem cells modified with CXCR4 to acute failing liver improves liver regeneration. World J Gastroenterol. 2014;20(40):14884-94.
15. He J, Wang C, Sun Y, et al. Exendin-4 protects bone marrow-derived mesenchymal stem cells against oxygen/glucose and serum deprivationinduced apoptosis through the activation of the CAMP/PKA signaling pathway and the attenuation of ER stress. Int J Mol Med. 2016;37(4):889-900.

16. Marte BM, Downward J. PKB/Akt: connecting phosphoinositide 3-kinase to cell survival and beyond. Trends Biochem Sci. 1997;22(9):355-8.

17. Bulj Z, Duchi S, Bevilacqua A, et al. Protein kinase B/AKT isoform 2 drives migration of human mesenchymal stem cells. Int J Oncol. 2013;42(1):118-26.

18. Gnecchi $\mathrm{M}, \mathrm{He} \mathrm{H}$, Noiseux $\mathrm{N}$, et al. Evidence supporting paracrine hypothesis for Akt-modified mesenchymal stem cell-mediated cardiac protection and functional improvement. FASEB J. 2006;20(6):661-9.

19. Gnecchi $\mathrm{M}, \mathrm{He} \mathrm{H}$, Liang OD, et al. Paracrine action accounts for marked protection of ischemic heart by Akt-modified mesenchymal stem cells. Nat Med. 2005;11(4):367-8

20. Mangi AA, Noiseux N, Kong D, et al. Mesenchymal stem cells modified with Akt prevent remodeling and restore performance of infarcted hearts. Nat Med. 2003;9(9):1195-201.

21. Croitoru-Lamoury J, Lamoury FM, Caristo M, et al. Interferon-gamma regulates the proliferation and differentiation of mesenchymal stem cells via activation of indoleamine 2,3 dioxygenase (IDO). PLoS One. 2011;6(2): e14698

22. Yang ZX, Mao GX, Zhang J, et al. IFN-gamma induces senescence-like characteristics in mouse bone marrow mesenchymal stem cells. Adv Clin Exp Med. 2017:26(2):201-6.

23. Li XL, Shang BX, Li YN, et al. IFNY and TNFa synergistically induce apoptosis of mesenchymal stem/stromal cells via the induction of nitric oxide. Stem Cell Res Ther. 2019;10(1):18. https://doi.org/10.1186/s13287-018-1102-z.

24. Ren G, Zhang L, Zhao X, et al. Mesenchymal stem cell-mediated immunosuppression occurs via concerted action of chemokines and nitric oxide. Cell Stem Cell. 2008;2(2):141-50.

25. Ren G, Zhao X, Zhang L, et al. Inflammatory cytokine-induced intercellular adhesion molecule-1 and vascular cell adhesion molecule-1 in mesenchymal stem cells are critical for immunosuppression. J Immunol. 2010;184(5):2321-8.

26. Ma S, Xie N, Li W, et al. Immunobiology of mesenchymal stem cells. Cell Death Differ. 2014;21(2):216-25

27. Soleymaninejadian E, Pramanik K, Samadian E. Immunomodulatory properties of mesenchymal stem cells: cytokines and factors. Am J Reprod Immunol. 2012;67(1):1-8.

28. Tiegs $\mathrm{G}$, Hentschel J, Wendel A. A T cell-dependent experimental liver injury in mice inducible by concanavalin A. J Clin Invest. 1992;90(1):196-203.

29. Gantner F, Leist M, Lohse AW, et al. Concanavalin A-induced T-cellmediated hepatic injury in mice: the role of tumor necrosis factor. Hepatology. 1995:21(1):190-8.

30. Kusters S, Gantner F, Kunstle $G$, et al. Interferon gamma plays a critical role in T cell-dependent liver injury in mice initiated by concanavalin A. Gastroenterology. 1996;111(2):462-71.

31. De Becker A, Riet IV. Homing and migration of mesenchymal stromal cells: How to improve the efficacy of cell therapy? World J Stem Cells. 2016;8(3): $73-87$.

32. Ricks DM, Kutner $R$, Zhang $X Y$, et al. Optimized lentiviral transduction of mouse bone marrow-derived mesenchymal stem cells. Stem Cells Dev. 2008:17(3):441-50.

33. Liu S, Lu W, Li S, et al. Identification of JL1037 as a novel, specific, reversible lysine-specific demethylase 1 inhibitor that induce apoptosis and autophagy of AML cells. Oncotarget. 2017;8(19):31901-14.

\section{Publisher's Note}

Springer Nature remains neutral with regard to jurisdictional claims in published maps and institutional affiliations. 